\title{
Crystal Engineering of Photoreactive and Photosalient Crystals
}

Jagadese J Vittal, Department of Chemistry, National University of Singapore, Singapore 117543, E-mail: chmjjv@nus.edu.sg

Mechanically responsive materials change their shape and size or move in space by light, thermal, pressure or chemical energy. Of these, dynamic molecular crystals undergo various movements like curling, crawling, jumping, leaping, hopping, popping, splitting, wiggling, and exploding, when exposed to heat (thermosalient effect) or light (photosalient effect). These photo-dynamic and thermaldynamic crystals create new ways of transforming light and heat energy into mechanical work. These effects are similar to popping of mustard seeds on hot oil and corn on hot surfaces. Usually anisotropic volume changes accompanied by the sudden release of the accumulated strain energy are responsible for the salient effects by heat and light. On the contrary, it was rather challenging to synthesize photoreactive solids that can undergo photodimerization reaction of metal complexes, coordination polymers (CPs) and metalorganic frameworks (MOFs), in the past. But it is now possible to design a reactive solid using the crystal engineering principles with ease. While investigating the [2+2] photoreactivity of crystals under UV light, we observed violent popping of single crystals of several metal complexes under UV light. In this talk we will discuss the design of some of these photoreactive crystals to exhibit photosalient behavior. 